\title{
Video Article \\ Evaluating the Differentiation Capacity of Mouse Prostate Epithelial Cells Using Organoid Culture
}

\author{
Preston D. Crowell ${ }^{*}$, Jenna M. Giafaglione* ${ }^{* 1}$, Takao Hashimoto ${ }^{2}$, Johnny A. Diaz ${ }^{2}$, Andrew S. Goldstein ${ }^{2,3,4,5,6}$ \\ ${ }^{1}$ Molecular Biology Interdepartmental Program, University of California, Los Angeles \\ ${ }^{2}$ Department of Molecular, Cell, and Developmental Biology, University of California, Los Angeles \\ ${ }^{3}$ Department of Urology, David Geffen School of Medicine, University of California, Los Angeles \\ ${ }^{4}$ Eli and Edythe Broad Center of Regenerative Medicine and Stem Cell Research, University of California, Los Angeles \\ 5 Jonsson Comprehensive Cancer Center, University of California, Los Angeles \\ ${ }^{6}$ Molecular Biology Institute, University of California, Los Angeles \\ *These authors contributed equally
}

Correspondence to: Andrew S. Goldstein at agoldstein@mednet.ucla.edu

URL: https://www.jove.com/video/60223

DOI: doi: $10.3791 / 60223$

Keywords: Cancer Research, Issue 153, organoid, prostate, epithelium, progenitor, basal, luminal, differentiation, mouse

Date Published: $11 / 22 / 2019$

Citation: Crowell, P.D., Giafaglione, J.M., Hashimoto, T., Diaz, J.A., Goldstein, A.S. Evaluating the Differentiation Capacity of Mouse Prostate Epithelial Cells Using Organoid Culture. J. Vis. Exp. (153), e60223, doi:10.3791/60223 (2019).

\section{Abstract}

The prostate epithelium is comprised predominantly of basal and luminal cells. In vivo lineage tracing has been utilized to define the differentiation capacity of mouse prostate basal and luminal cells during development, tissue-regeneration and transformation. However, evaluating cell-intrinsic and extrinsic regulators of prostate epithelial differentiation capacity using a lineage tracing approach often requires extensive breeding and can be cost-prohibitive. In the prostate organoid assay, basal and luminal cells generate prostatic epithelium ex vivo. Importantly, primary epithelial cells can be isolated from mice of any genetic background or mice treated with any number of small molecules prior to, or after, plating into three-dimensional (3D) culture. Sufficient material for evaluation of differentiation capacity is generated after 7-10 days. Collection of basal-derived and luminal-derived organoids for (1) protein analysis by Western blot and (2) immunohistochemical analysis of intact organoids by whole-mount confocal microscopy enables researchers to evaluate the ex vivo differentiation capacity of prostate epithelial cells. When used in combination, these two approaches provide complementary information about the differentiation capacity of prostate basal and luminal cells in response to genetic or pharmacological manipulation.

\section{Video Link}

The video component of this article can be found at https://www.jove.com/video/60223/

\section{Introduction}

Basal and luminal cells comprise the majority of the prostate epithelium ${ }^{1}$. Lineage tracing studies have revealed that these cell types are predominantly self-sustained by distinct progenitors in the adult mouse ${ }^{2}$; however, luminal differentiation from basal progenitors has been observed in several contexts including development ${ }^{3,4}$, tissue regeneration ${ }^{5}$, inflammation ${ }^{6,7}$ and prostate cancer initiation ${ }^{2,8}$. Furthermore, emerging data supports the existence of multipotent luminal progenitors as well as luminal-committed progenitors ${ }^{9}$. In metastatic prostate cancer, differentiation from an AR-dependent luminal lineage to an AR-indifferent lineage with basal and neuroendocrine features represents an increasingly appreciated mechanism of resistance to androgen pathway inhibitors ${ }^{10,11,12}$. Therefore, as differentiation is implicated in normal physiology, cancer initiation and resistance to therapy, elucidating key molecular regulators of prostate epithelial cell differentiation is critical.

The mouse prostate organoid model has emerged as an elegant ex vivo context to study prostate epithelial cell differentiation ${ }^{9,13,14}$. In this assay, individual epithelial cells are plated into a 3D matrix where they generate glandular structures containing both basal and luminal cells within 1 week. While existing approaches for plating cells into organoid culture can be used to efficiently generate organoids, these approaches require further optimization ${ }^{14}$. Notable challenges associated with culturing prostate organoids include (1) excluding two-dimensional (2D) colonies that form beneath the Matrigel (matrix gel) from analysis, (2) maintaining the integrity of the matrix gel during media changes, and (3) counting organoids accurately. This paper outlines an approach to generate organoids from epithelial cells isolated from mouse prostate. The approach described entails coating plates with poly(2-hydroxyethyl methacrylate) (Poly-HEMA) to prevent the occurrence of 2D colonies. Furthermore, cells are plated into a matrix gel ring, rather than a matrix gel disc, which makes changing the media and counting organoids less challenging. These techniques allow researchers to more easily investigate how genetic alterations or small molecules introduced prior to, or during, organoid formation alter key processes such as differentiation.

Harvesting of prostate organoids for Western blot or immunohistochemical analysis by whole-mount confocal microscopy can provide valuable mechanistic insight into differentiation ${ }^{13}$, yet well-established protocols to prepare organoids for such techniques are lacking. This manuscript describes approaches to harvest organoids for (1) collection of protein lysate, or (2) fixation and staining for confocal microscopy. Importantly, 
the approach described for fixing and staining prostate organoids is considerably improved in relation to existing methods. While these rely on sectioning organoids ${ }^{15}$, the method described in this manuscript utilizes intact organoids, which helps protect against organoid damage during sample preparation. When used in combination, Western blot and confocal microscopy can provide valuable insight into the molecular regulators of differentiation. Alternatively, these approaches can be used to model other processes such as development and transformation.

Protocol

All methods described here have been approved by the Institutional Review Board at the University of California, Los Angeles.

NOTE: A schematic illustrating the approaches described in the paper is provided in Figure 1.

\title{
1. Isolating Mouse Basal and Luminal Prostate Epithelial Cells Using Fluorescence- activated Cell Sorting (FACS) - TIMING: 30 Min
}

\author{
NOTE: Perform steps $1.3-1.5$ in the dark.
}

1. After dissociating cells from total mouse prostate as described in Lawson et al. ${ }^{16}$, transfer the cells to FACS tubes and resuspend $0.1-5 \times 10^{6}$ cells in $100 \mu \mathrm{L}$ of dissociation media (Table 1).

2. Add the appropriate volume of the following directly-conjugated primary antibodies: CD45, CD31, Ter-119, EpCAM and CD49f.

3. Incubate on ice, protected from light, for $20 \mathrm{~min}$.

NOTE: It is recommended to utilize $10 \%$ of the total dissociated cells for unstained and single-stained controls. These controls are necessary to set the correct compensation and voltage for sorting.

4. Quench antibody cocktail by adding $1 \mathrm{~mL}$ of dissociation media to each sample. Pellet the cells by centrifugation at $800 \times \mathrm{g}$ for $5 \mathrm{~min}$ at room temperature (RT) and remove the supernatant by aspirating.

5. Resuspend the cells in appropriate volume $\left(250 \mu \mathrm{L}\right.$ per $1 \times 10^{6}$ cells $)$ of dissociation media containing $1 \mu \mathrm{g} / \mathrm{mL} 4^{\prime}, 6$-diamidino-2-phenylindole (DAPI). Proceed to FACS. Flow cytometry plots demonstrating isolation of mouse basal and luminal prostate epithelial cells are illustrated in Figure 2.

\section{Plating Sorted Prostate Epithelial Cells into Primary Mouse Organoid Culture - TIMING: 2-3 H (Excluding Poly-HEMA-coated Plate Preparation)}

NOTE: Plates are coated with Poly-HEMA to prevent 2D colony formation on the surface of the well beneath the matrix gel. Prepare Poly-HEMAcoated plates 1 day prior to plating sorted basal or luminal prostate epithelial cells into mouse organoid culture. Thaw $1 \mathrm{~mL}$ aliquots of reduced growth factor matrix gel, hereafter referred to as matrix gel, on ice $2 \mathrm{~h}$ prior to step 2.1. Y-27632 (ROCK inhibitor) should be added to mouse organoid media immediately prior to step 2.1. Perform steps $2.1-2.8$ on ice.

1. Pellet the cells in $5 \mathrm{~mL}$ round-bottom tubes by centrifugation at $800 \times g$ for 5 min at $4{ }^{\circ} \mathrm{C}$ and aspirate the supernatant.

2. Wash the cell pellet in $500 \mu \mathrm{L}$ of mouse organoid media (Table 2$)^{14}$.

3. Pellet the cells by centrifugation at $800 \times g$ for $5 \mathrm{~min}$ at $4{ }^{\circ} \mathrm{C}$ and aspirate the supernatant.

4. Resuspend in mouse organoid media at a cell density of 1,000 cells $/ \mu \mathrm{L}$.

5. To prepare master mixes, mix epithelial cells suspended in mouse organoid media with matrix gel to generate a final mixture that contains $25 \%$ cells/media and $75 \%$ matrix gel. Basal cells are typically plated at a concentration of $100-2,000$ cells $/ 80 \mu \mathrm{L}$, whereas luminal cells are typically plated at a concentration of $2,000-10,000$ cells $/ 80 \mu \mathrm{L}$. The density of cells plated varies depending upon the day of anticipated material collection, and the desired downstream application.

NOTE: Chill appropriately sized tube(s) for expected master mix volume 5 min prior to master mix preparation. To ensure the matrix gel does not harden while handling, it is critical to chill the pipette tip by pipetting the matrix gel 3-4 times prior to transferring it to a new tube.

6. Add $80 \mu \mathrm{L}$ of the matrix gel/cell mixture per well of a 24-well plate. Pipetting a droplet onto the lower half of the wall of the well, while avoiding direct contact with the Poly-HEMA coating is recommended. After adding the matrix gel, swirl the plate to allow the matrix gel/cell mixture to form a ring around the rim of the well.

7. Place the 24-well plate into a $37^{\circ} \mathrm{C} 5 \% \mathrm{CO}_{2}$ incubator right-side up for $10 \mathrm{~min}$ to allow the matrix gel to partially harden. NOTE: Begin warming mouse organoid media at $37^{\circ} \mathrm{C}$ immediately after placing the 24-well plate in the incubator.

8. After incubating for $10 \mathrm{~min}$, flip the 24 -well plate upside-down and incubate for an additional 50 min to allow the matrix gel to completely harden.

9. Add $350 \mu \mathrm{L}$ of pre-warmed mouse organoid media dropwise to the center of each well. NOTE: To maintain the integrity of the matrix gel, it is critical to avoid the matrix gel ring while adding media.

10. After adding the media, return the 24 -well plate to the $37^{\circ} \mathrm{C} 5 \% \mathrm{CO}_{2}$ incubator.

\section{Replenishing Mouse Organoid Media - TIMING: 10-15 Min Per 24-well Plate}

NOTE: Existing media should be replaced with fresh media every $48 \mathrm{~h}$. Before each media change, pre-warm mouse organoid media. It is not necessary to add ROCK inhibitor to the media used for replenishing.

1. Tilt the 24-well plate at a $45^{\circ}$ angle and gently remove existing media from the center of each well using a p1000 pipette, while avoiding the matrix gel ring.

2. Add $350 \mu \mathrm{L}$ of pre-warmed mouse organoid media as in step 2.9. It is recommended to add a larger volume of media (up to $1 \mathrm{~mL}$ ) to organoids cultured for longer than 5 days in order to prevent rapid depletion of key nutrients and growth factors. 


\section{Extracting Protein Lysate from Prostate Organoids for Western Blot Analysis - TIMING:} 2.5-4 H

NOTE: Prior to collecting organoids for protein lysate extraction, prepare and pre-warm dispase-containing media (Table 1).

1. Remove the media from each well as in step 3.1.

2. To collect organoids, repeatedly blast the matrix gel by pipetting $1 \mathrm{~mL}$ of dispase-containing media directly onto the matrix gel ring until the entire ring is dislodged, and transfer to a $1.5 \mathrm{~mL}$ microcentrifuge tube.

NOTE: It is critical to avoid direct contact with the Poly-HEMA-coated wells. Direct contact may cause contamination of the collected material with Poly-HEMA, which could negatively impact cell survival.

3. Place the $1.5 \mathrm{~mL}$ microcentrifuge tube(s) into a $37{ }^{\circ} \mathrm{C} 5 \% \mathrm{CO}_{2}$ incubator for $30 \mathrm{~min}$ to $1 \mathrm{~h}$ to allow complete digestion of the matrix gel by dispase.

4. Pellet organoids by centrifugation at $800 \times g$ for $5 \mathrm{~min}$ at RT and remove the supernatant using a micropipette.

5. Add phosphate-buffered saline (PBS) to the organoid pellet and resuspend by gently flicking

NOTE: Failure to sufficiently resuspend the organoid pellet may result in the contamination of organoid material with residual dispase or matrix gel.

6. Pellet the organoids by centrifugation at $800 \times g$ for $5 \mathrm{~min}$ at RT and remove the supernatant using a micropipette

7. Fast freeze the organoid pellets by placing each tube into a solution containing dry ice and methanol. Store the tube(s) until future use at -80 ${ }^{\circ} \mathrm{C}$. Alternatively, extract protein lysate immediately following step 4.6.

8. Resuspend the organoid pellets in $100 \mu \mathrm{L}$ of protein lysis buffer (Table 1) per $10 \mu \mathrm{L}$ of packed cell volume. Flick to resuspend. NOTE: If resuming after fast-freezing, ensure protein lysis buffer is thawed prior to removing samples from $-80^{\circ} \mathrm{C}$, as lysis buffer must be added to samples immediately in order to prevent phosphatase and protease activity.

9. Incubate the samples in protein lysis buffer on ice for at least $45 \mathrm{~min}$.

NOTE: It is recommended to sonicate prior to incubation on ice to increase the efficiency of nuclear protein recovery; however, sonication is not required. If sonication is not performed, proceed to step 4.10 .

1. To sonicate, submerge tubes in wet ice and gently apply the tip of the sonic dismembrator to the outside of the microcentrifuge tube. Sonicate for $40 \mathrm{~s}$ at $20 \mathrm{kHz}$.

10. Proceed to Western blot following established protocols.

\section{Fixing and Staining Prostate Organoids for Immunohistochemical Analysis by Whole- mount Confocal Microscopy}

1. Collecting prostate organoids from 24-well plates - TIMING: 45-60 $\mathrm{min}$

NOTE: When collecting prostate organoids to process for confocal microscopy, it is critical to handle them with care in order to maintain their structure. The collection protocol below is designed to reduce disruption of organoid structure during isolation.

1. Remove the media from each well as in step 3.1.

2. Digest the matrix gel by incubating with $500 \mu \mathrm{L}$ of dispase-containing media (Table 1) for 30 min in a $37{ }^{\circ} \mathrm{C} 5 \% \mathrm{CO}_{2}$ incubator.

3. Collect digested organoid suspension in a microcentrifuge tube and pellet the organoids by centrifugation at $800 \times g$ for 3 min at RT. Remove the supernatant.

2. Whole-mount immunofluorescent staining of prostate organoids - TIMING: 3-4 days (1-5 h/day)

1. Add $500 \mu \mathrm{L}$ of $4 \%$ paraformaldehyde in PBS and incubate for $2 \mathrm{~h}$ at RT with gentle shaking.

2. Pellet the organoids by centrifugation at $800 \times g$ for $3 \mathrm{~min}$ at RT, remove the supernatant, and wash the pellet with $1 \mathrm{~mL}$ of PBS for 15 min with gentle shaking.

3. Wash the pellet as in step 5.2.2 for additional two times.

4. Pellet the organoids by centrifugation at $800 \times g$ for 3 min at RT and remove the supernatant. Add $1 \mu \mathrm{g} / \mathrm{mL}$ DAPI in blocking solution (Table 1). Incubate for $2 \mathrm{~h}$ at RT or alternatively overnight at $4{ }^{\circ} \mathrm{C}$ with gentle shaking.

5. Pellet the organoids by centrifugation at $800 \times g$ for $3 \mathrm{~min}$ at RT and remove the supernatant. Add primary antibody (rabbit anti-p63, mouse anti-cytokeratin 8) in blocking solution and incubate overnight at $4{ }^{\circ} \mathrm{C}$ with gentle shaking.

6. Pellet the organoids by centrifugation at $800 \times g$ for 3 min at RT and remove the supernatant. Wash the pellet with $1 \mathrm{~mL}$ of PBS for 15 min with gentle shaking.

7. Wash the pellet as in step 5.2.6 for additional two times.

8. Pellet the organoids by centrifugation at $800 \times g$ for $3 \mathrm{~min}$ at RT and remove the supernatant. Add secondary antibody (goat anti-rabbit IgG-Alexa Fluor 594, goat anti-mouse IgG-Alexa Fluor 488) in blocking solution and incubate overnight at $4{ }^{\circ} \mathrm{C}$ with gentle shaking.

9. Pellet the organoids by centrifugation at $800 \times g$ for $3 \mathrm{~min}$ at RT, remove the supernatant, and wash the pellet with $1 \mathrm{~mL}$ of PBS for 15 min with gentle shaking.

10. Wash the pellet as in step 5.2.9 for additional two times.

\section{Tissue Clearing and Mounting of the Stained Prostate Organoids for Whole-mount Confocal Microscopy - TIMING: 7 H}

1. Pellet the organoids by centrifugation at $800 \times g$ for 3 min at RT and remove the supernatant

2. Add $1 \mathrm{~mL}$ of $30 \%$ sucrose in PBS with $1 \%$ Triton $\mathrm{X}-100$ and incubate for $2 \mathrm{~h}$ at RT with gentle shaking.

3. Pellet the organoids by centrifugation at $800 \times g$ for 3 min at RT and remove the supernatant.

4. Add $1 \mathrm{~mL}$ of $45 \%$ sucrose in PBS with $1 \%$ Triton $\mathrm{X}-100$ and incubate for $2 \mathrm{~h}$ at RT with gentle shaking. 
5. Pellet the organoids by centrifugation at $800 \times g$ for $3 \mathrm{~min}$ at RT and remove the supernatant.

6. Add $1 \mathrm{~mL}$ of $60 \%$ sucrose in PBS with $1 \%$ Triton $\mathrm{X}-100$ and incubate for $2 \mathrm{~h}$ at $\mathrm{RT}$ with gentle shaking.

7. Pellet the organoids by centrifugation at $800 \times g$ for $3 \mathrm{~min}$ at RT and remove $95 \%$ of the supernatant.

NOTE: The pellet becomes looser as the concentration of sucrose becomes higher. Observing the DAPI-stained organoids under the UV light to confirm that they were not lost during removal of the supernatant is recommended.

8. Transfer a $10-20 \mu \mathrm{L}$ droplet of the remaining suspension to a chambered coverslip and proceed to confocal microscopy. NOTE: Coverslip fragments can be placed on either side of the droplet to be used as spacers (Figure 4C). These prevent organoids from collapsing when a coverslip is placed over the droplet.

\section{Representative Results}

Prostate epithelial cells are plated into mouse organoid culture where they form organoids, which are harvested prior to preparation for downstream analysis (Figure 1).

Basal and luminal epithelial cells are isolated using FACS. After excluding DAPI ${ }^{+}$cells and depleting Lin $^{+}$cells (CD45, CD31, Ter119), basal and luminal cells are distinguished based on differential expression of EpCAM and CD49f (Figure 2). The approach described to plate prostate basal and luminal cells into organoid culture entails: (1) plating cells into matrix gel rings, and (2) coating wells with Poly-HEMA. Plating into rings has been previously described in Agarwal et al ${ }^{9}$. Utilizing this approach (Figure 3A) allows researchers to more easily avoid the matrix gel while replenishing the media (Step 3), and more easily count organoids by following the circumference of the well. Coating wells with Poly-HEMA has been shown to prevent 2D colony formation in retinal organoids ${ }^{17}$; however, this approach has not been utilized in the prostate organoid model. Importantly, coating wells with Poly-HEMA (Table 3) eliminates the occurrence of 2D colonies without interfering with organoid formation (Figure 3B). These modifications expand the capabilities of the prostate organoid assay.

Basal and luminal cells form organoids with distinct morphologies (Figure 4A). While most basal-derived organoids are similar in size (100-300 $\mu \mathrm{m}$ diameter) after 7 days in culture, luminal-derived organoids exhibit significant heterogeneity ( $30-450 \mu \mathrm{m}$ diameter). Furthermore, most basal-derived organoids contain lumens surrounded by multi-layered epithelium (Figure 4A, top), whereas luminal-derived organoids range in morphology from hollow, with single-layered epithelium to solid, with multi-layered cords of cells that do not canalize (Figure 4A, bottom). The approaches described above to prepare organoids for downstream analysis (Steps 4,5), were used to investigate whether these phenotypic differences are reflective of differences in lineage marker expression. Western blot analysis revealed that basal and luminal-derived organoids retain features associated with basal and luminal primary cells. Basal-derived organoids express higher levels of the basal marker cytokeratin 5 (K5), whereas luminal-derived organoids express higher levels of the luminal marker cytokeratin 8 (K8) (Figure 4B). Both basal and luminal markers were detected in basal and luminal-derived organoids in the bulk population, perhaps suggestive of differentiation (Figure 4B).

We sought to characterize lineage marker expression in basal-derived organoids and determine whether morphologically distinct luminal-derived organoids exhibit differences in marker expression by staining intact organoids and performing confocal microscopy (Figure 4D). Basal-derived organoids contained multi-layered epithelium with outer layers expressing high levels of the basal marker p63 and moderate levels of the luminal marker K8 (p63 $\left.3^{\text {hi }}, \mathrm{K}^{\mathrm{mid}}\right)$, and inner layers without detectable levels of p63 and high levels of K8 (p63 $\left.{ }^{\mathrm{lo}}, \mathrm{K} 8^{\mathrm{hi}}\right)$ (Figure 4D, top). While all cells in single-layered luminal-derived organoids stained positively for $\mathrm{K} 8$, only select cells contained nuclear p63 (Figure 4D, bottom). These data validate the approaches to harvest and prepare organoids for analysis by Western blot or confocal microscopy and thereby expand the capability of the organoid assay to study key cellular processes, including differentiation. 


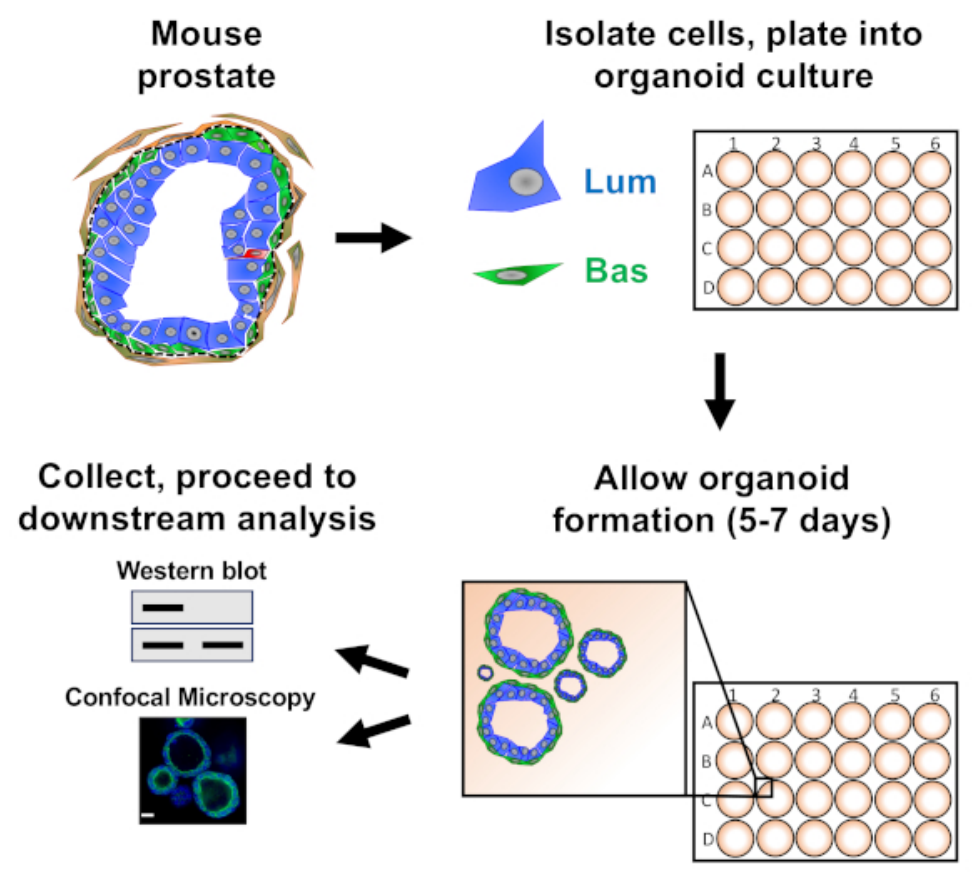

Figure 1: Schematic illustrating workflow to generate prostate organoids for collection and analysis. Total mouse prostate is dissociated and basal and luminal prostate epithelial cells are isolated by fluorescence-activated cell sorting via established protocols ${ }^{8,19}$. Basal or luminal cells suspended in a mixture of mouse organoid media and matrix gel are plated into matrix gel rings. After 5 to 7 days of culture, organoids are harvested for analysis by Western blot or confocal microscopy. Please click here to view a larger version of this figure.
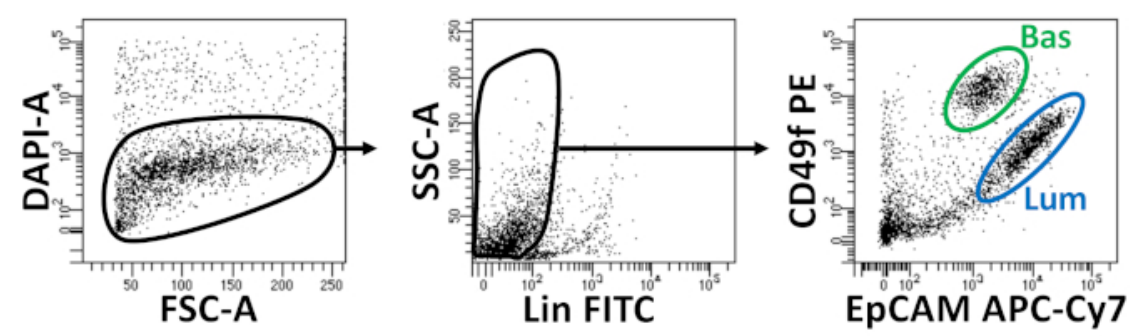

Figure 2: Isolation of mouse basal and luminal prostate epithelial cells using fluorescence-activated cell sorting (FACS). Dissociated cells from mouse prostate are stained with DAPI, to distinguish live from dead cells, and surface antibodies, to distinguish basal from luminal cells, prior to FACS. Left $=$ Gated on DAPI- cells. FSC-A $=$ forward-scatter. Center $=$ Gated on Lin ${ }^{-}$cells $\left(C D 45^{10}, C D 31^{10}\right.$, Ter119 $\left.9^{\mathrm{lo}}\right) . S S C-A=$ side-scatter. Right = Basal cells $($ Bas $)\left(\right.$ EpCAM $\left.^{\mathrm{hi}}, \mathrm{CD}_{4} \mathrm{f}^{\mathrm{hi}}\right)$, Luminal cells (Lum) $\left(\mathrm{EpCAM}^{\mathrm{hi}}, \mathrm{CD} 49 \mathrm{f}^{\mathrm{mid}}\right)$. Please click here to view a larger version of this figure. 
A.

Representative well

(24-well plate)

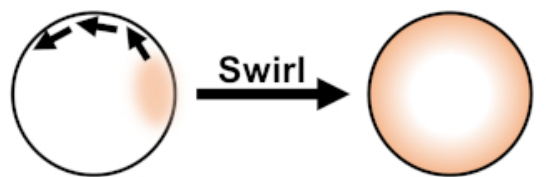

Matrigel Droplet Matrigel Ring

B.

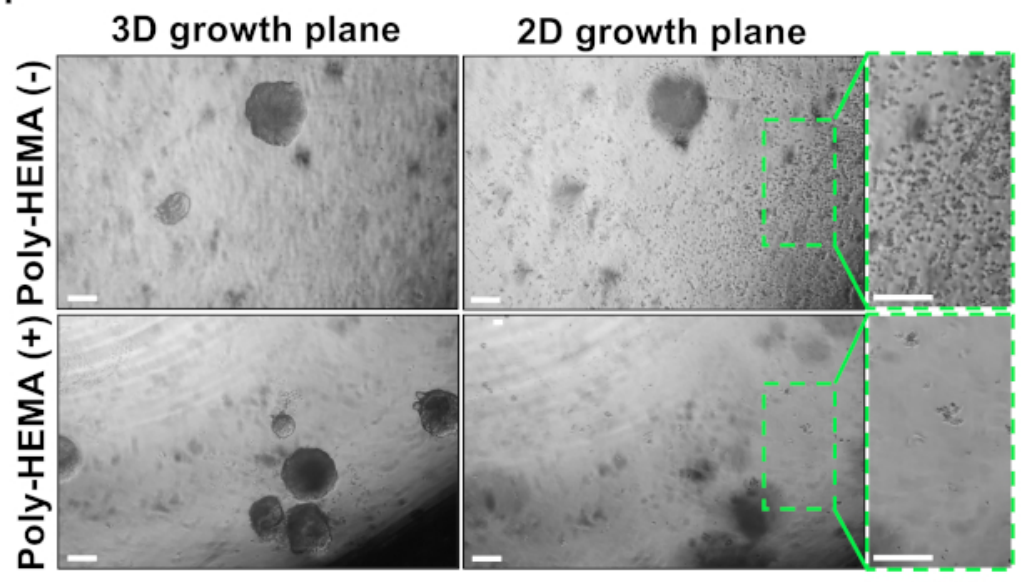

Figure 3: Establishment of mouse prostate organoids. (A) Schematic illustrating approach to generate a matrix gel ring in a well of a 24-well plate. (B) Representative phase contrast images of organoids (3D growth plane) and two-dimensional colonies (2D growth plane) formed 7 days after plating prostate epithelial cells into un-coated (Poly-HEMA (-)), or coated (Poly-HEMA (+)) 24-well plates. Boxed regions within 2D growth plane are magnified on the right. Scale bars $=200 \mu \mathrm{m}$. Please click here to view a larger version of this figure. 
A.

\section{Primary Organoids}

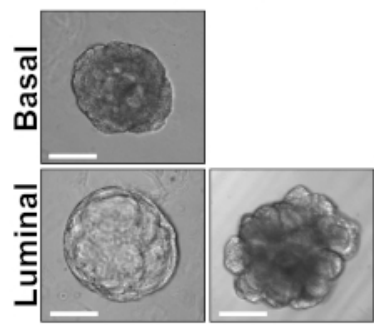

B.

Primary Organoids

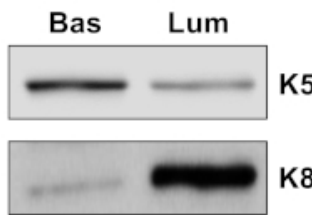

$\mathrm{K} 8$

$\mathrm{HH} 3$

C.

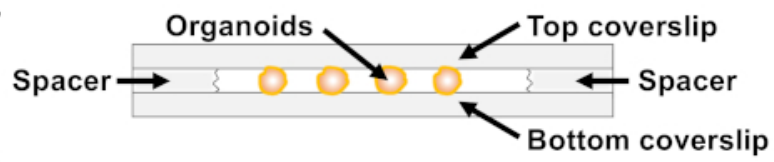

D.

p63

K8

DAPI

Merge

DIC

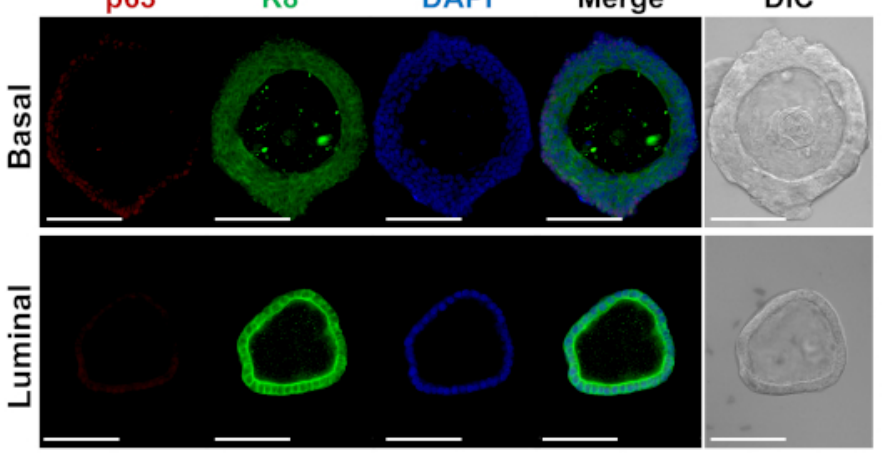

Figure 4: Analysis of lineage marker expression in prostate organoids by Western blot and whole-mount confocal microscopy. (A) Representative phase contrast images of basal-derived (top), and luminal-derived (bottom) organoids after 7 days of culture. Scale bar $=100$ $\mu \mathrm{m}$. (B) Western blot analysis of basal-derived (Bas) and luminal-derived (Lum) organoids after 5 days of culture. Staining for the basal marker, cytokeratin 5 (K5), and the luminal marker, cytokeratin 8 (K8), and a loading control, histone $\mathrm{H} 3(\mathrm{HH} 3)$. (C) Schematic illustrating chambered coverslip with spacers. (D) Representative differential interference contrast (DIC) and immunofluorescent images of basal-derived (top) and luminal-derived (bottom) organoids after 7 days of culture. Staining for p63 (red), K8 (green) and DAPI (blue) individually and merged. Scale bars $=100 \mu \mathrm{m}$. Please click here to view a larger version of this figure.

\begin{tabular}{|l|l|}
\hline Recipes & \multicolumn{2}{l|}{$\begin{array}{l}\mathrm{mg} / \mathrm{mL} \text { dispase }+10 \mu \mathrm{M} \text { ROCK inhibitor in advanced DMEM F12. } \\
\text { Filter sterilize using } 0.22 \mu \mathrm{m} \text { filter. }\end{array}$} \\
\hline Dispase-containing media & $\begin{array}{l}10 \% \text { FBS }+1 \times \text { Penicillin-Streptomycin in RPMI 1640. Filter sterilize } \\
\text { using } 0.22 \mu \mathrm{m} \text { filter. }\end{array}$ \\
\hline Protein lysis buffer & RIPA buffer + phosphatase inhibitors + protease inhibitors \\
\hline Blocking solution & $10 \%$ FBS in PBS with $0.2 \%$ Triton X-100 \\
\hline
\end{tabular}

Table 1: Instructions for the preparation of key solutions. 


\begin{tabular}{|l|l|}
\hline Component & Concentration \\
\hline B-27 & $1 \times$ (dilute from 50x concentrate) \\
\hline GlutaMAX & $1 \times$ (dilute from 100x concentrate) \\
\hline N-acetyl-L-cysteine & $1.25 \mathrm{mM}$ \\
\hline Normocin & $50 \mu \mathrm{g} / \mathrm{mL}$ \\
\hline Recombinant Human EGF, Animal-Free & $50 \mathrm{ng} / \mathrm{mL}$ \\
\hline Recombinant Human Noggin & $100 \mathrm{ng} / \mathrm{mL}$ \\
\hline R-spondin 1-conditioned media & $10 \%$ conditioned media \\
\hline A83-01 & $200 \mathrm{nM}$ \\
\hline DHT & $1 \mathrm{nM}$ \\
\hline Y-27632 dihydrochloride (ROCK inhibitor) & $10 \mu \mathrm{M}$ \\
\hline Advanced DMEM/F-12 & Base media \\
\hline $\begin{array}{l}\text { R-spondin 1-conditioned media is generated as described in Drost, et al. } \\
\text { using } 0.22 \mu \text {. After addition of all components, filter sterilize mouse organoid media }\end{array}$ \\
\hline
\end{tabular}

Table 2: Instructions for the preparation of mouse organoid media.

\begin{tabular}{|c|c|}
\hline \multicolumn{2}{|c|}{ Protocol for preparing Poly-HEMA-coated plates } \\
\hline 1 & $\begin{array}{l}\text { Add } 0.25 \mathrm{~g} \text { Poly-HEMA to } 50 \mathrm{~mL} 98 \% \text { EtOH. Dissolve Poly-HEMA at } 37 \\
{ }^{\circ} \mathrm{C} \text { on a shaker. This process takes at least } 4 \mathrm{~h} \text {. }\end{array}$ \\
\hline 2 & Filter sterilize Poly-HEMA using $0.22 \mu \mathrm{m}$ filter. \\
\hline 3 & Add $200 \mu \mathrm{l}$ of Poly-HEMA solution per well of a 24 -well plate(s). \\
\hline 4 & $\begin{array}{l}\text { Remove lid(s) from 24-well plate(s) after adding Poly-HEMA and allow } \\
\text { solution to evaporate overnight. }\end{array}$ \\
\hline 5 & $\begin{array}{l}\text { Wash each well twice with PBS and ensure wells are completely dry } \\
\text { prior to storage after final wash. NOTE: Disrupting the Poly-HEMA } \\
\text { coating during washing could contribute to 2-dimensional growth upon } \\
\text { plating epithelial cells into organoid culture. To prevent damage to } \\
\text { Poly-HEMA-coated wells, avoid direct contact with the pipette tip while } \\
\text { washing. The integrity of the Poly-HEMA-coated wells will remain intact } \\
\text { unless the Poly-HEMA is scraped off by the pipette tip. }\end{array}$ \\
\hline 6 & $\begin{array}{l}\text { Poly-HEMA-coated plates can be stored at } 4{ }^{\circ} \mathrm{C} \text { for up to two weeks. } \\
\text { NOTE: Wrapping plates in parafilm prior to storage will reduce the risk of } \\
\text { contamination. }\end{array}$ \\
\hline
\end{tabular}

Table 3: Protocol for preparation of Poly-HEMA-coated plates.

\section{Discussion}

Prostate epithelial cell differentiation has been implicated in both normal prostate biology $y^{2,3,4,5,6,7}$ and disease biology ${ }^{8,10,11,12}$; however, the master regulators of this process remain undefined. Identifying key regulators of prostate epithelial cell differentiation has been difficult in part due to the absence of well-established contexts to model it. While 2D monolayer culture can be used to model differentiation ${ }^{11,12}$, this context fails to recapitulate the complex prostate microenvironment. Furthermore, in vivo contexts to model differentiation do not lend themselves to mechanistic studies, as they are challenging to manipulate. Therefore, the identification of an easy to manipulate, yet physiologically-relevant context, to study differentiation is critical.

The prostate organoid model represents an elegant ex vivo context where basal to luminal differentiation is reported to occur. Methods to establish prostate organoids are well established ${ }^{14}$; however, further optimization of these methods is necessary. Furthermore, approaches to harvest and prepare prostate organoids for analysis are not clearly described. This paper describes an approach to plate prostate epithelial cells isolated from mouse prostate into organoid culture. This approach allows researchers to (1) prevent the occurrence of 2D colonies during organoid formation, (2) reduce the risk of disruption to the matrix gel during media replenishment, and (3) count organoids more effectively. In addition, this manuscript outlines approaches to harvest organoids for preparation for Western blot analysis, or whole-mount confocal microscopy. Importantly, the approach utilized to prepare organoids for confocal microscopy maintains the intact structure of organoids through its duration, which reduces organoid damage prior to image acquisition. Altogether, the approaches described expand the capabilities of the prostate organoid assay.

Notably, the organoid-forming capacity of basal and luminal cells can be altered both by methods used to isolate the respective populations, and by culture conditions. The organoid culture conditions used in this assay were first described by Karthaus et al. ${ }^{13}$. Whereas Karthaus et al. have reported that basal cells have a higher organoid forming capacity $(15 \%)$ than luminal cells $(1 \%)^{13}$, Chua et al., using distinct isolation methods and culture conditions, have reported that luminal cells $(0.2-0.3 \%)$ have a higher organoid-forming capacity than basal cells $(0.03 \%)^{20}$. Overall, 
methods described by Karthaus et al. lead to higher organoid-forming rates for both basal and luminal cells, likely reflecting differences in the approach used to isolate basal and luminal cells ${ }^{13}$, as opposed to culture conditions that bias against organoid formation from luminal cells. It remains unclear whether the protocol described in this manuscript favors luminal organoid formation from multipotent luminal progenitors, or committed-luminal progenitors ${ }^{9}$. Though timely and cost-prohibitive, in vivo lineage tracing studies can be used to validate progenitor features associated with distinct prostate epithelial lineages elucidated in the organoid assay.

Processes such as development, differentiation and transformation are not only relevant to prostate biology, but also relevant to the biology of other tissues including the brain, lung, intestine, pancreas and liver. The methods described facilitate the utilization of the organoid model to study these processes in not only the prostate, but also a wide range of tissues.

\section{Disclosures}

The authors have nothing to disclose.

\section{Acknowledgments}

PDC and JMG are supported by the Ruth L. Kirschstein National Research Service Award GM007185. JAD is supported by the National Institute of General Medical Sciences of the National Institutes of Health (R25GM055052) awarded to T. Hasson and the Saul Martinez Scholarship. ASG is supported by the Spitzer Family Foundation Fund and the Gill Endowment. This work was supported by the American Cancer Society (RSG-17-068-01-TBG), Department of Defense (W81XWH-13-1-0470), Margaret E. Early Medical Research Trust, NIH/NCl (P50CA092131/ UCLA SPORE in Prostate Cancer), Rose Hills Foundation, and support from UCLA's Jonsson Comprehensive Cancer Center, Broad Stem Cell Research Center, Clinical and Translational Science Institute, and Institute of Urologic Oncology.

\section{References}

1. Kwon, O.J., Xin, L. Prostate epithelial stem and progenitor cells. American Journal of Clinical and Experimental Urology. 2 (3), 209-218, (2014).

2. Choi, N., Zhang, B., Zhang, L., Ittmann, M., Xin, L. Adult Murine Prostate Basal and Luminal Cells Are Self-Sustained Lineages that Can Both Serve as Targets for Prostate Cancer Initiation. Cancer Cell. 21 (2), 253-265, (2012).

3. Ousset, M., Van Keymeulen, A., et al. Multipotent and unipotent progenitors contribute to prostate postnatal development. Nature Cell Biology. 14 (11), 1131-1138, (2012).

4. Wang, J., et al. Symmetrical and asymmetrical division analysis provides evidence for a hierarchy of prostate epithelial cell lineages. Nature Communications. 5, 1-13, (2014).

5. Wang, Z.A., Mitrofanova, A., et al. Lineage analysis of basal epithelial cells reveals their unexpected plasticity and supports a cell-of-origin model for prostate cancer heterogeneity. Nature Cell Biology. 15, (3), 274-283, (2013).

6. Kwon, O.J., Zhang, B., Zhang, L., Xin, L. High fat diet promotes prostatic basal-to-luminal differentiation and accelerates initiation of prostate epithelial hyperplasia originated from basal cells. Stem Cell Research. 16 (3), 682-691, (2016).

7. Kwon O.J., Zhang L, Ittmann, M.M., Xin L. Prostatic inflammation enhances basal-to-luminal differentiation and accelerates initiation of prostate cancer with a basal cell origin. Proceedings of the National Academy of Sciences. 192 (3), 997-999, (2014).

8. Stoyanova, T., et al. Prostate cancer originating in basal cells progresses to adenocarcinoma propagated by luminal-like cells. Proceedings of the National Academy of Sciences. 110 (50), 20111-20116, (2013).

9. Agarwal, S., Hynes, P.G., et al. Identification of Different Classes of Luminal Progenitor Cells within Prostate Tumors. Cell Reports. 13 (10), 2147-2158, (2015).

10. $\mathrm{Ku}, \mathrm{S} . \mathrm{Y}$., et al. Rb1 and Trp53 cooperate to suppress prostate cancer lineage plasticity, metastasis, and antiandrogen resistance. Science. 355 (6320), 78-83, (2017).

11. Mu, P., et al. SOX2 promotes lineage plasticity and antiandrogen resistance in TP53- and RB1-deficient prostate cancer. Science. 355 (6320), 84-88, (2017).

12. Bishop, J.L., et al. The Master Neural Transcription Factor BRN2 Is an Androgen Receptor-Suppressed Driver of Neuroendocrine Differentiation in Prostate Cancer. Cancer Discovery. 7 (1), 54-71, (2016).

13. Karthaus, W.R., et al. Identification of multipotent luminal progenitor cells in human prostate organoid cultures. Cell. 159 (1), 163-175, (2014).

14. Drost, J., Karthaus, W.R., et al. Organoid culture systems for prostate epithelial and cancer tissue. Nature Protocols. 11 (2), 347-358, (2016).

15. McCray, T., Richards, Z., Marsili, J., Prins, G.S., Nonn, L. Handling and Assessment of Human Primary Prostate Organoid Culture. Journal of Visualized Experiments. (143) (2019).

16. Lawson, D.A, Xin, L., Lukacs, R.U., Cheng, D., \& Witte, O.N. Isolation and functional characterization of murine prostate stem cells. Proceedings of the National Academy of Sciences of the United States of America. 104 (1), 181-186, (2007).

17. Chen, H.Y., Kaya, K.D., Dong, L., \& Swaroop, A. Three-dimensional retinal organoids from mouse pluripotent stem cells mimic in vivo development with enhanced stratification and rod photoreceptor differentiation. Molecular vision. 22, 1077-1094, (2016).

18. Liu, X., et al. Low CD38 Identifies Progenitor-like Inflammation-Associated Luminal Cells that Can Initiate Human Prostate Cancer and Predict Poor Outcome. Cell Reports. 17 (10), 2596-2606, (2016).

19. Lukacs, R.U., Goldstein, A.S., Lawson, D.A., Cheng, D., Witte, O.N. Isolation, cultivation and characterization of adult murine prostate stem cells. Nature protocols. 5 (4), 702-713, (2010).

20. Chua, C. W., Shibata, M., et al. Single luminal epithelial progenitors can generate prostate organoids in culture. Nature Cell Biology. 16 (10), 951-961, (2014). 